\title{
Validation and implementation of telephone-administered version of the Multidimensional Prognostic Index (TELE-MPI) for remote monitoring of community-dwelling older adults
}

\author{
Carlo Custodero ${ }^{1} \cdot$ Barbara Senesi $^{2} \cdot$ Alessandra Pinna $^{2} \cdot$ Annarosa Floris $^{2} \cdot$ Martina Vigo $^{2} \cdot$ Marcella Fama $^{2} \cdot$ \\ Valeria Mastropierro $^{1} \cdot$ Carlo Sabbà $^{1} \cdot$ Camilla Prete $^{2} \cdot$ Alberto Pilotto $^{1,2} \mathbb{C}$
}

Received: 25 February 2021 / Accepted: 22 April 2021 / Published online: 18 May 2021

(c) The Author(s), under exclusive licence to Springer Nature Switzerland AG 2021

\begin{abstract}
Background During the recent lockdown measures adopted by national authorities to contain the COVID-19 pandemic, many vulnerable older patients with chronic conditions, normally followed in ambulatory setting, needed to be monitored and managed in alternative ways, including telemedicine.

Aims In the framework of a telemedicine program, we aimed to validate and implement a telephone-administered version of the Multidimensional Prognostic Index (TELE-MPI) among community-dwelling older outpatients.

Method From March 9 to May 11, 2020, 131 older patients (82.1 years; 74\% females) were interviewed using a telephonebased survey to calculate the TELE-MPI. The standard MPI was performed face-to-face three months apart. The BlandAltman methodology measured the agreement between the two tools. Multivariate logistic regression models were built to ascertain the prognostic value of TELE-MPI and TELE-MPI classes (low, moderate, or severe risk) on negative outcomes occurring during the lockdown period.

Results Mean MPI and TELE-MPI values were 0.523 and 0.522 , respectively. Lower and upper $95 \%$ limits of agreement were -0.122 and +0.124 , respectively, with only $4.6 \%$ of observations outside the limits. Each 0.1 increase of TELE-MPI score was significantly correlated with higher incidence of psychiatric disorders [odd ratio (OR): 1.57; 95\% confidence interval (CI) $1.27,1.95$ ] and falls (OR: 1.41; 95\% CI 1.08, 1.82) in community-dwelling-older adults.

Discussion TELE-MPI showed a strong agreement with the standard MPI and was able to predict psychiatric disorders and falls during lockdown period.
\end{abstract}

Conclusion TELE-MPI may represent a useful way to follow by remote the health status of older adults.

Keywords Telemedicine · Multidimensional Prognostic Index · Comprehensive Geriatric Assessment (CGA) · COVID19 $\cdot$ Psychiatric disorders $\cdot$ Falls

\section{Introduction}

The World Health Organization (WHO) defines telemedicine as the application of innovative information and communication technologies, in order to guarantee the provision of

Alberto Pilotto

alberto.pilotto@galliera.it

1 Department of Interdisciplinary Medicine, Clinica Medica e Geriatria "Cesare Frugoni”, University of Bari Aldo Moro, Bari, Italy

2 Department Geriatric Care, Orthogeriatrics and Rehabilitation, E.O. Ospedali Galliera, Via Mura delle Cappuccine 16, 35121 Genova, Italy more efficient healthcare services, especially in situations where the distance between involved subjects plays a decisive role [1]. The most common user interfaces in telemedicine include desktop and laptop computers, fax machines, regular and mobile phones, video cameras and a variety of "stand alone" systems.

Telemedicine can be applied in the healthcare for secondary prevention in people with chronic diseases to reduce the risk of possible complications; for the diagnosis in order to support the conventional approach; for the treatment to follow the patient's response; and for rehabilitation [2]. Telemedicine, in particular, can play a key role in the management of the frail older adults, who not only need more frequent monitoring, but at the same time present increasing 
difficulties in access to healthcare facilities [3-5]. Older adults have shown interest and adaptability to use of technology [4]. Furthermore, the use of telemedicine in contexts without assistance services is at least as effective as the faceto-face visit [3].

The sudden and pervasive diffusion of the COVID-19 pandemic resulted in a serious emergency situation throughout the social and welfare sectors dealing with the management of frail older adults [6-8]. Most of the resources have been converted for the emergency; most of the services and home assistance, which the older patients benefited from, have been suspended. The social restrictions, imposed during the lockdown, had a substantial impact on the management of outpatient activities with cancellations or postponements of visits and consults. Moreover, more vulnerable patients, such as frail older adults, are at higher risk for psychiatric problems and adverse events related to social isolation [9] and when infected with COVID-19 reported an increased risk of morbidity, admission to intensive care units and death [6].

In this context, telemedicine can offer a practical method for monitoring community-dwelling, frail older adults encouraging the adoption of new modalities of medical care $[10,11]$. However, there is also a need of prognostic tools able to early identify frail older adults more at risk of dismal outcomes not merely related to COVID-19 infections. The Multidimensional Prognostic Index derived from Comprehensive Geriatric Assessment (CGA) has been demonstrated as a feasible and reliable tool predicting morbidity, mortality, hospitalization, institutionalization in community-dwelling older adults [12]. Moreover, it offers the opportunity to guide the healthcare providers in clinical decision making and allocation of resources [13].

We aimed to validate a telephone-administered version of the MPI (TELE-MPI) among community-dwelling older outpatients and then testing the usefulness of this tool in predicting adverse outcomes such as occurrence of psychiatric symptoms, falls, hospitalization and death during the COVID-19 related lockdown.

\section{Methods}

\section{Study population}

Between March 9th 2020 and May 11th 2020, 170 patients usually followed in ambulatory setting at the E.O. Galliera Hospital of Genova, Italy, were contacted to undergo a televisit by a multi-professional team consisting of geriatrician, nurse and neuropsychologist. Of these, 160 responded and 131 subjects, aged from 55 to 95 years, accepted an interaction via pc/tablet or smartphone, using a dedicated connection (Jiitsimeet/Galliera). Subjects were included whether:
(1) attended the outpatient clinics of the Galliera Hospital, (2) lived in community, (3) provided an informed consent or had the availability of a proxy for informed consent, (4) were willing to participate in the study and (5) had both complete standard MPI and TELE-MPI. No specific age criterion was applied, but only three patients were younger than 65 years old. This study was performed in accordance with the ethical standards in the 1964 Declaration of Helsinki and received formal ethical approval by Ethical Committee of Department of Education of the University of Genoa (DISFOR), Genoa, Italy.

Information about occurrence of COVID-19 manifestations (e.g. fever $\geq 38{ }^{\circ} \mathrm{C}$, cough, dyspnea and other symptoms), falls, call to emergency medical service, access to the emergency department and death were collected. Presence of caregiver during the lockdown was also assessed. Diagnosis of psychiatric disorders (i.e. depression, anxiety, sleep disorder, delirium) was formulated according the American Psychiatric Association's Diagnostic and Statistical Manual fifth edition (DSM-5).

\section{Multidimensional prognostic index (MPI)}

The standard MPI was performed face-to-face by health professionals three months apart (before or after the lockdown) from the telephone interview. The MPI was calculated with the information from eight different domains of the CGA as previously reported:

1. Functional status was evaluated through the Katz's activities of daily living (ADL) index, which defines the level of independence in six daily personal care activities (bathing, toileting, feeding, dressing, continence and transferring in and out of bed or chair) [14].

2. Independence was measured by means of the Lawton's instrumental activities of daily living (IADL) scale, which assesses independence in eight activities that are more cognitively and physically demanding than the ADL, that is, managing finances, using a telephone, taking medications, shopping, using transportation, preparing meals, doing housework and washing [15].

3. Cognitive status was determined through the Short Portable Mental Status Questionnaire (SPMSQ), a 10-item questionnaire investigating orientation, memory, attention, calculation and language [16].

4. Comorbidity was examined using the cumulative illness rating scale (CIRS), a five-point ordinal scale (score $1-5)$ to estimate the severity of pathology in each of 13 systems, including cardiac, vascular, respiratory, eye-ear-nose-throat, upper and lower gastrointestinal, hepatic, renal, genitourinary, musculoskeletal, skin, nervous system, endocrine-metabolic and psychiatric disorders. Based on the ratings, the comorbidity index 
(CIRS-CI) score, which reflects the number of concomitant diseases, was derived from the total number of categories in which moderate or severe levels (grade from 3 to 5) of disease were identified (range from 0 to 13) [17].

5. Nutritional status was investigated with the Mini Nutritional Assessment Short Form (MNA-SF), which includes information on (1) anthropometric measures (body mass index and weight loss), (2) neuropsychiatric problems and recent psychiatric stress, (3) mobility and (4) decline in food intake [18].

6. Risk of developing pressure sores was evaluated through the Exton-Smith Scale (ESS), a five-item questionnaire determining physical and mental condition, activity, mobility and incontinence [19]. For each item, a score from 1 to 4 is assigned.

7. Medication use was defined according to the Anatomical Therapeutics Chemical Classification code system (ATC classification) and the number of drugs used by patients was recorded. Patients were defined as being on medication if they were taking any of the drugs included in the ATC classification at the moment of study inclusion.

8. Cohabitation status included living alone, in an institution, or with family members.

For each domain, was assigned a score: $0=$ no problems, $0.5=$ minor problems and $1=$ major problems, based on conventional cutoff points derived from literature for the singular items. The sum of the scores of each domain was divided by 8 to obtain a final MPI risk score ranging from $0=$ no risk to $1=$ higher risk of mortality.

\section{Telephone-administered MPI (TELE-MPI)}

Similarly to the MPI domains, the TELE-MPI considered the following eight areas:

1. Functional status assessed through the ADL scale [14].

2. Mobility assessed inquiring about the abilities to transfer from bed to chair or wheelchair, walking and going up and down the stairs [20]. The ability of doing these activities was categorized as yes (1 point) or no (0 point). We opted for this scale rather than ESS, for evaluation of mobility, because the dichotomous choice allows an easier judgment by patient or caregiver compared to ESS.

3. Independence using the Lawton's IADL scale, as reported earlier [15].

4. Cognitive status with SPMSQ scale [16].

5. Nutritional status investigated with the MNA-SF [18].

6. Number of medications.

7. Comorbidity: CIRS comorbidity is the number of health problems/diseases that are so severe to require chronic drug therapies in 13 aspects of health [17]. Therefore, CIRS can be self-reported listing health conditions that require medications for their treatment.

8. Cohabitation status as previously stated.

As for the MPI, a tripartite hierarchy was used for each item and described in Table 1, according to previously reported cutoff values [21]. For the assessment of mobility, which is the only domain evaluated in different way from the standard MPI, we used the following, previously proposed, cutoff values: score equal to 3 or $2=0$; score equal to $1=0.5$, score equal to $0=1$ [20]. The sum of the scores assigned to each domain was divided by 8 to obtain a final TELE-MPI risk score ranging from $0=$ no risk to $1=$ high
Table 1 Domains of the telephone-administered-MPI and its calculation

\begin{tabular}{llll}
\hline TELE-MPI & \multicolumn{2}{l}{ Level of risk } & \\
\cline { 2 - 4 } & Risk low $=0$ & Risk moderate $=0.5$ & $2-0$ \\
\hline 1. ADL & $6-5$ & $4-3$ & 0 \\
2. MOBILITY & $3-2$ & 1 & $3-0$ \\
3. IADL & $8-6$ & $5-4$ & $8-10$ \\
4. SPMSQ & $0-3$ & $4-7$ & $7-0$ \\
5. MNA-SF & $14-12$ & $11-8$ & $\geq 3$ \\
6. Self-reported CIRS & 0 & $1-2$ & $\geq 7$ \\
7. Number of drugs & $0-3$ & $4-6$ & Alone \\
8. Cohabitation status & Family & Nursing home & \\
Sum the numbers assigned to each domain and divide by 8 & Total score TELE-MPI &
\end{tabular}

$A D L$ activities of daily living, CIRS Cumulative Illness Rating Scale, IADL Instrumental Activities of daily Living, MNA-SF Mini Nutritional Assessment Short Form, SPMSQ Short Portable Mental Status Questionnaire, TELE-MPI Telephone-administered Multidimensional Prognostic Index 
risk of mortality. Also, according to the previously established MPI categories [22], the TELE-MPI was expressed as three grades of risk: TELE-MPI-1 low risk (values $\leq 0.33$ ), TELE-MPI-2 moderate risk (values between 0.34 and 0.66 ) and TELE-MPI-3 high risk (MPI value $>0.66$ ).

\section{Statistical analysis}

Descriptive statistics were expressed as absolute and relative frequencies (percent) for categorical variables and as mean and standard deviation (SD) for continuous variables. The difference between the TELE-MPI and the standard MPI was tested with the $t$ test for paired samples. We verified the agreement between the TELE-MPI and the standard MPI using the Bland-Altman plot (BAP) methodology [23]. This methodology provides an objective measure of agreement (95\% limits of agreement) and a visual representation of the level of the overlap of two different scores (plot of the difference vs. the average of the two scores) [23].

Bivariate logistic regressions were performed to test the association between TELE-MPI and different negative outcomes including onset of psychiatric disorders, falls, call to the emergency medical service, access to the emergency department and death. Multivariable logistic regression models were developed in order to identify whether TELE-MPI, expressed both as continuous variable (per 0.1 increase of the score) and categorical variable (TELEMPI-1, TELE-MPI-2, TELE-MPI-3), was an independent predictor of negative outcomes taking into account potential confounding factors such as age, gender, presence of caregiver during the lockdown period. A two-tailed significance level at $p=0.05$ was set for each test. All the analyses were performed using SPSS v26.0 software for Windows (SPSS, Chicago, IL, USA).

\section{Results}

Table 2 summarizes the characteristics of the studied cohort. We enrolled 131 community-dwelling older adults (74\% females) with mean (SD) age of 82.1 years (6.6). Half of participants lived alone (50.4\%) and only 23 subjects (17.6\%) were assisted by a caregiver during lockdown.

Mean (SD) MPI and TELE-MPI scores were 0.523 $(0.223)$ and $0.522(0.222)$ respectively (Table 2$)$. The mean difference (SD) between the MPI and the TELE-MPI was equal to $+0.001(0.063)$ (Table 2). Lower and upper 95\% limits of agreement were respectively equal to -0.122 and +0.124 . Only 6 of 131 observations (4.6\%) fell outside the limits of agreement (Fig. 1). Among participants the perceived acceptability of telemedicine program was high (89.3\%). The majority of participants (60.3\%) did not report
Table 2 Characteristics of the study population

\begin{tabular}{ll}
\hline Age, mean (SD) & $82.1(6.6)$ \\
Female, $n(\%)$ & $97(74)$ \\
Caregiver, $n(\%)$ & $42(32)$ \\
Caregiver during lockdown, $n(\%)$ & $23(17.6)$ \\
Influenza vaccination, $n(\%)$ & $95(72.5)$ \\
Pneumococcal vaccination, $n(\%)$ & $11(8.4)$ \\
COVID-19 manifestation, $n(\%)$ & $52(39.7)$ \\
Psychiatric disorders, $n(\%)$ & $58(44.3)$ \\
Falls, $n(\%)$ & $33(25.2)$ \\
Call to the emergency medical service, $n(\%)$ & $14(10.7)$ \\
Access to the emergency department, $n(\%)$ & $13(9.9)$ \\
Mortality, $n(\%)$ & $1(0.8)$ \\
Therapeutic change, $n(\%)$ & $35(26.7)$ \\
Acceptability of telemedicine, $n(\%)$ & $117(89.3)$ \\
MPI, mean (SD) & $0.523(0.223)$ \\
TELE-MPI, mean (SD) & $0.522(0.222)$ \\
Difference MPI - TELE-MPI, mean (SD) & $+0.001(0.063)$ \\
\hline
\end{tabular}

MPI Multidimensional prognostic index, $S D$ standard deviation, TELE-MPI Telephone-administered MPI

signs and symptoms related to COVID-19 infection. Most frequent adverse events were represented by new onset psychiatric disorders $(44.3 \%)$ and falls (25.2\%). In 35 cases (26.7\%) a therapeutic change was suggested. Incidence of hospitalization and death was relatively low: $9.9 \%$ and $0.8 \%$, respectively.

\section{Prognostic value of TELE-MPI on negative outcomes}

According the TELE-MPI class $27.5 \%$ subjects were distributed in the low-risk category (TELE-MPI-1), $42 \%$ in the moderate risk (TELE-MPI-2) and 30.5\% in the high-risk category (TELE-MPI-3). We explored potential association between TELE-MPI and negative outcomes occurring during the COVID-19 pandemic including onset of psychiatric disorders, falls, call to the emergency medical service, access to the emergency department and death. Among these selected outcomes, the TELE-MPI score (as well as the standard MPI) was significantly associated only with the occurrence of psychiatric disorders $(p<0.0001)$ and with falls $(p=0.001)$.

In a multivariable logistic regression model, each increase of 0.1 point in the TELE-MPI predicted an increase of about $60 \%$ of psychiatric disorders risk [odd ratio (OR) 1.57; $95 \%$ confidence interval (CI) 1.27, 1.95; $p<0.0001]$ independently by other potential confounders such as age, gender and presence of caregiver even during the lockdown period. In particular, as compared to patients in the lowest risk class (TELE-MPI-1), those in the highest risk class (TELEMPI-3) approximately had a tenfold higher risk of developing psychiatric disorders (OR: 10.36; 95\% CI 3.06, 35.10; 
Fig. 1 Bland-Altman plot of agreement between MPI and TELE-MPI

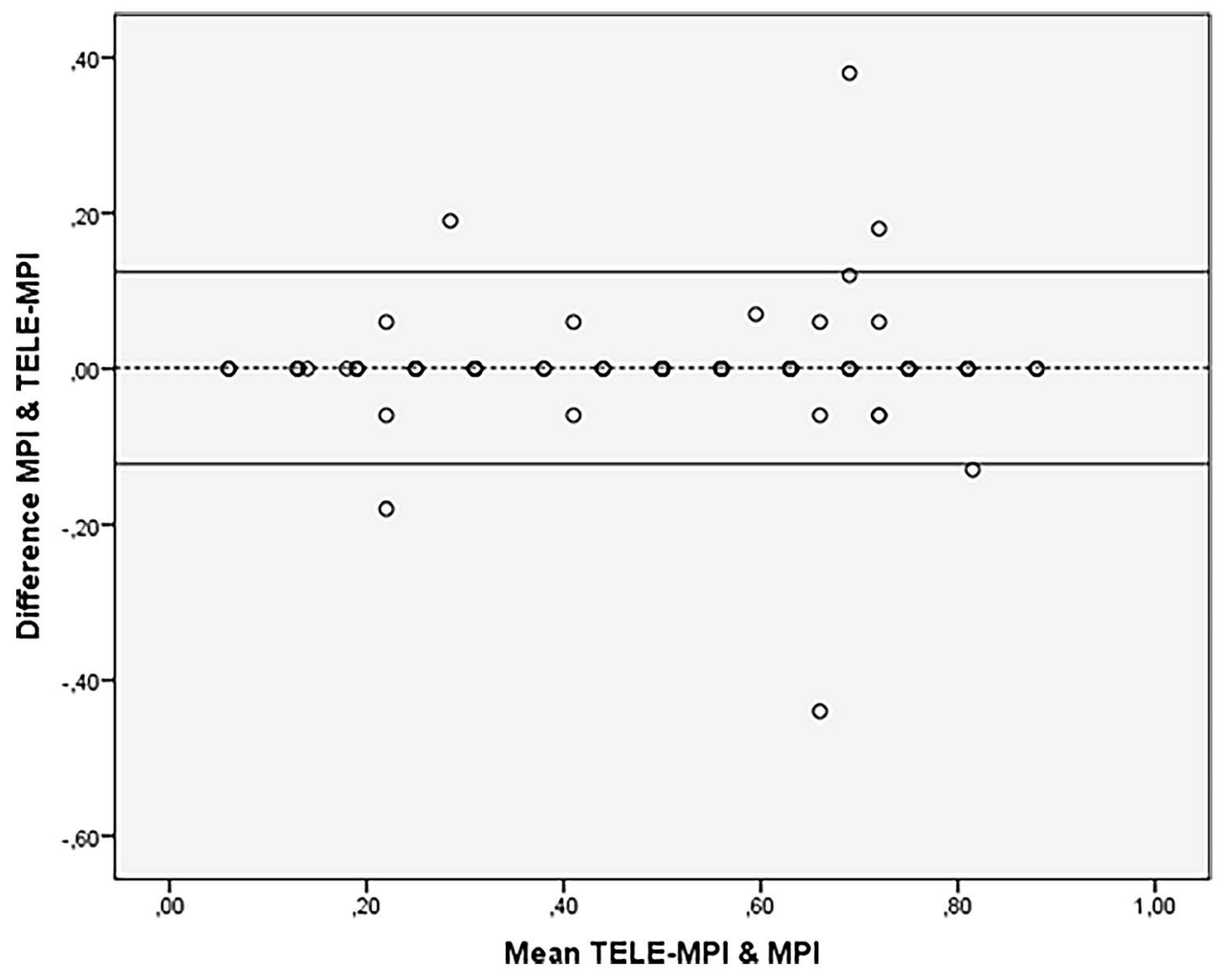

Table 3 Logistic model for prediction of psychiatric disorders and falls by TELE-MPI as continuous scale or stratified in risk classes

\begin{tabular}{lllc}
\hline & Odds ratio & $95 \%$ CI & $p$ value \\
\hline Psychiatric disorders $^{\mathrm{a}}$ & & & \\
TELE-MPI $(\times 0.1$ increase) & 1.57 & $1.27-1.95$ & $<0.0001$ \\
TELE-MPI class & & & \\
$\quad$ ( (Low risk) & 1.0 (REF) & & \\
2 (Moderate risk) & 5.14 & $1.70-15.57$ & 0.04 \\
3 (High risk) & 10.36 & $3.06-35.10$ & $<0.0001$ \\
Falls ${ }^{\mathrm{b}}$ & & & \\
TELE-MPI $(\times 0.1$ increase) & 1.41 & $1.08-1.82$ & 0.01 \\
TELE-MPI class & & & \\
1 (Low risk) & $1.0(\mathrm{REF})$ & & \\
2 (Moderate risk) & 3.24 & $0.77-13.63$ & 0.11 \\
3 (High risk) & 4.95 & $1.07-22.82$ & 0.04 \\
\hline
\end{tabular}

CI Confidence Interval, TELE-MPI Telephone-administered Multidimensional Prognostic Index

${ }^{a}$ Model adjusted for age, gender and presence of a caregiver during lockdown

${ }^{\mathrm{b}}$ Model adjusted for age, gender, presence of a caregiver during lockdown and occurrence of psychiatric disorders

$p<0.0001$ ) and those in the moderate risk class (TELEMPI-2) about five times higher risk (OR: 5.14; 95\% CI 1.70, 15.57; $p=0.004$ ) (Table 3).

After adjusting for age, gender, presence of a caregiver during lockdown and new onset psychiatric disorders, an increase of 0.1 point in the TELE-MPI corresponded to a $41 \%$ increased probability of falls (OR: $1.41 ; 95 \%$ CI 1.08 , $1.82 ; p=0.01)$ independently. Compared to patients in the lowest risk class (TELE-MPI-1), those in the highest risk class (TELE-MPI-3) had roughly five times higher risk of developing falls (OR: 4.95; 95\% CI 1.07, 22.82; $p=0.04$ ) (Table 3). Furthermore, patients with new onset psychiatric disorders had a twofold higher risk of falls (OR: 2.52; $95 \%$ CI 1.03, 6.15; $p=0.043$ ) (data not shown).

\section{Discussion}

In the present study we found that telephone-administered version of MPI (TELE-MPI) has strong agreement with traditional MPI tool performed face-to-face, in communitydwelling older adults. We found that higher TELE-MPI score is an independent predictor of new psychiatric disorders and falls during the lockdown period related to COVID19 pandemic. Compared to subjects in the lowest risk category based on TELE-MPI stratification (TELE-MPI-1), those in the highest risk category (TELE-MPI-3) had 10and 5-time more pronounced risk of psychiatric disorders and falls occurrence, respectively.

Older adults with chronic conditions represent a high-risk category not only for their vulnerability to the SARS-CoV-2 [6], but also for the potential short and long-term consequences correlated to the suspension of healthcare support 
and the breakdown of social networks $[8,9]$. Social isolation and loneliness are well-known risk factors for morbidity and mortality among older adults [24]. Telemedicine programs may offer the possibility to deal with these aspects related to the social distancing $[10,25]$. In the last months, several initiative focused on telehealth in community-dwelling older adults have been set up, also solicited by national health authorities, to offer adequate support to this frail population [26, 27]. For example, Beauchet et al. developed a telephone screening test named "Evaluation SOcioGERiatrique" (ESOGER) which included assessment of: (1) COVID-19 manifestation; (2) frailty; (3) psychological distress; (4) social isolation and (5) caregivers' burden, to early detect homebound older adults at risk of negative outcomes [26]. However, this tool still lacks validation studies.

To the best of our knowledge, TELE-MPI is the first prognostic tool for community-dwelling older adults validated using a telephone interview. In contrast with previous evidence on MPI [12], in this cohort, both MPI and TELE-MPI did not show predictive accuracy for death and access to the emergency department, but this might be explained by limited sample size, relatively short follow-up, different type of setting and potentially by the effect itself of telemedicine program. Previous studies showed that standard MPI is also an independent predictor of depressive disorders and falls $[28,29]$. In the Irish Longitudinal Study on Ageing, carried out on 1,854 older adults followed-up over two years, higher baseline MPI values were significantly associated with incident depressive symptoms (highest MPI tertile vs. lowest: OR: 2.96; 95\% CI 1.50-5.85) [28]. Evidence from Osteoarthritis Initiative study in 885 community-dwelling older people with knee osteoarthritis or at high risk for this condition showed that subjects in high-risk MPI category (MPI-3) had significantly higher risk of recurrent falls compared to those in MPI-1 category (OR: 5.98; 95\% CI 3.29-10.86) during 8-year follow-up [29]. The present findings may suggest that the telephone-administered version of MPI could have similar efficacy of standard MPI tool in predicting risk of psychiatric disorders and falls.

This study has some limitations. Firstly, the sample size is relatively small thus may be underpowered on some outcomes as hospitalization and mortality. Secondly, the crosssectional design does not allow to establish a causal-effect relationship between multidimensional impairment assessed by TELE-MPI and occurrence of negative outcomes. Thirdly, the MPI score could change over time, however it is quite reasonable that in a population of community-dwelling the MPI variations in such limited time frame would be low relevant. Finally our study population is constituted by patients who agreed to participate in the telemedicine program and thus could have access and familiarity with technologies. Therefore, present findings might not be generalizable to the broader population of community-dwelling older adults. Future longitudinal studies with larger population are warranted to test usefulness of TELE-MPI in risk stratification and clinical decision making.

However, this study has also some important strengths. Indeed, we validated for the first time a telephone-administered version of a well-established prognostic tools as the MPI. Besides the current utilization, the TELE-MPI might have several further applications in clinical practice including monitoring post-hospitalization course or effectiveness of home-based interventions in older adults. This approach may be sustainable for the patients and less time-consuming for the healthcare providers. More televisits can be scheduled in the same time spent for just one face-to-face, meaning more often follow-ups and more strict control of patients' conditions.

In conclusion, our study suggests that TELE-MPI was a feasible and reliable tool for remote monitoring of community-dwelling older people. TELE-MPI values can predict risk of psychiatric disorders and falls offering the opportunity to take appropriate preventive strategies.

Author contributions AP conceived and designed the study; $\mathrm{CC}$ wrote the original draft, performed the statistical analysis and takes responsibility of the accuracy of data analysis; VM assisted in data interpretation and wrote the manuscript; $\mathrm{BS}, \mathrm{AP}, \mathrm{AF}, \mathrm{MV}$ and $\mathrm{MF}$ contributed in data collection and literature search; CS, CP and AP critically revised the final manuscript. All authors approved the final draft submitted.

Funding This study was supported in part by a grant of the SIGOT (Società Italiana Geriatria Ospedale e Territorio) and by the Project Prestige co-funded by the Fondazione CARIGE. The contents of this article are the sole responsibility of the authors and can under no circumstances be ragarded as reflecting the position of the co-funders.

Availability of data and material The dataset is available from the corresponding author on reasonable request.

\section{Declarations}

Conflict of interest The authors have no conflicts of interest to declare that are relevant to the content of this article.

Ethics approval The Ethical Committee of Department of Education of the University of Genoa (DISFOR), Genoa, Italy approved the present study on 5 September 2019; study number 030 .

Consent to participate/publication Informed consent was obtained from all individual participants included in the study or from legal guardians.

\section{References}

1. World Health Organization (2010) Telemedicine: opportunities and developments in member states: report on the second 
Global survey on eHealth. Global observatory for eHealth series, vol 2. World Health Organization, Geneva

2. Guler NF, Ubeyli ED (2002) Theory and applications of telemedicine. J Med Syst 26:199-220. https://doi.org/10.1023/a: 1015010316958

3. Batsis JA, DiMilia PR, Seo LM et al (2019) Effectiveness of ambulatory telemedicine care in older adults: a systematic review. J Am Geriatr Soc 67:1737-1749. https://doi.org/10. 1111/jgs. 15959

4. Greenwald P, Stern ME, Clark S et al (2018) Older adults and technology: in telehealth, they may not be who you think they are. Int J Emerg Med 11:2. https://doi.org/10.1186/ s12245-017-0162-7

5. Betkus G, Freeman S, Martin-Khan M et al (2020) Comparison of in-person and telegeriatric follow-up consultations. J Telemed Telecare. https://doi.org/10.1177/1357633X20965416

6. Onder G, Rezza G, Brusaferro S (2020) Case-fatality rate and characteristics of patients dying in relation to COVID-19 in Italy. JAMA 323:1775-1776. https://doi.org/10.1001/jama.2020.4683

7. Lithander FE, Neumann S, Tenison E et al (2020) COVID-19 in older people: a rapid clinical review. Age Ageing 49(4):501-515. https://doi.org/10.1093/ageing/afaa093

8. Polidori MC, Maggi S, Mattace-Raso F et al (2020) The unavoidable costs of frailty: a geriatric perspective in the time of COVID19. Geriatric Care 6:8989. https://doi.org/10.4081/gc.2020.8989

9. Cudjoe TKM, Kotwal AA (2020) "Social Distancing" Amid a Crisis in Social Isolation and Loneliness. J Am Geriatr Soc 68(6):E27-E29

10. Hollander JE, Carr BG (2020) Virtually perfect? Telemedicine for Covid-19. N Engl J Med 382:1679-1681. https://doi.org/10.1056/ NEJMp2003539

11. Smith AC, Thomas E, Snoswell CL et al (2020) Telehealth for global emergencies: implications for coronavirus disease 2019 (COVID-19). J Telemed Telecare 26:309-313. https://doi.org/10. 1177/1357633X20916567

12. Pilotto A, Custodero C, Maggi S et al (2020) A multidimensional approach to frailty in older people. Ageing Res Rev 60:101047. https://doi.org/10.1016/j.arr.2020.101047

13. Cruz-Jentoft AJ, Daragjati J, Fratiglioni L et al (2020) Using the Multidimensional Prognostic Index (MPI) to improve costeffectiveness of interventions in multimorbid frail older persons: results and final recommendations from the MPI_AGE European Project. Aging Clin Exp Res 32:861-868. https://doi.org/10.1007/ s40520-020-01516-0

14. Katz S, Downs TD, Cash HR, Grotz RC (1970) Progress in development of the index of ADL. Gerontologist 10(1):20-30. https:// doi.org/10.1093/geront/10.1_part_1.20

15. Lawton MP, Brody EM (1969) Assessment of older people: selfmaintaining and instrumental activities of daily living. Gerontologist 9:179-186

16. Pfeiffer E (1975) A short portable mental status questionnaire for the assessment of organic brain deficit in elderly patients. $\mathrm{J}$ Am Geriatr Soc 23:433-441. https://doi.org/10.1111/j.1532-5415. 1975.tb00927.x

17. Linn BS, Linn MW, Gurel L (1968) Cumulative illness rating scale. J Am Geriatr Soc 16:622-626. https://doi.org/10.1111/j. 1532-5415.1968.tb02103.x
18. Rubenstein LZ, Harker JO, Salva A et al (2001) Screening for undernutrition in geriatric practice: developing the short-form mini-nutritional assessment (MNA-SF). J Gerontol A Biol Sci Med Sci 56:M366-372. https://doi.org/10.1093/gerona/56.6.m366

19. Bliss MR, McLaren R, Exton-Smith AN (1966) Mattresses for preventing pressure sores in geriatric patients. Mon Bull Minist Health Public Health Lab Serv 25:238-268

20. Pilotto A, Veronese N, Siri G et al (2020) Association between the multidimensional prognostic index and mortality over 15 years of follow-up in the inchianti study. J Gerontol A Biol Sci Med Sci. https://doi.org/10.1093/gerona/glaa237

21. Sancarlo D, D’Onofrio G, Franceschi M et al (2011) Validation of a modified-multidimensional prognostic index (m-MPI) including the mini nutritional assessment short-form (MNA-SF) for the prediction of one-year mortality in hospitalized elderly patients. J Nutr Health Aging 15:169-173. https://doi.org/10. 1007/s12603-010-0293-5

22. Pilotto A, Ferrucci L, Franceschi M et al (2008) Development and validation of a multidimensional prognostic index for one-year mortality from comprehensive geriatric assessment in hospitalized older patients. Rejuvenation Res 11:151-161. https://doi.org/10. 1089/rej.2007.0569

23. Bland JM, Altman DG (1986) Statistical methods for assessing agreement between two methods of clinical measurement. Lancet $1: 307-310$

24. Andrew MK, Mitnitski AB, Rockwood K (2008) Social vulnerability, frailty and mortality in elderly people. PLOS ONE 3:e2232. https://doi.org/10.1371/journal.pone.0002232

25. Kruse C, Fohn J, Wilson N et al (2020) Utilization barriers and medical outcomes commensurate with the use of telehealth among older adults: systematic review. JMIR Med Inform 8:e20359. https://doi.org/10.2196/20359

26. Beauchet O, Cooper-Brown L, Ivensky V et al (2020) Telemedicine for housebound older persons during the Covid-19 pandemic. Maturitas 142:8-10

27. Tan LF, Ho Wen Teng V, Seetharaman SK et al (2020) Facilitating telehealth for older adults during the COVID-19 pandemic and beyond: strategies from a Singapore geriatric center. Geriatr Gerontol Int 20:993-995. https://doi.org/10.1111/ggi.14017

28. Veronese N, Koyanagi A, Smith L et al (2020) Relationship between multidimensional prognostic index and incident depressive symptoms in older people: findings from the Irish longitudinal study on ageing. Int J Geriatr Psychiatry. https://doi.org/10. 1002/gps.5331

29. Veronese N, Siri G, Cella A et al (2020) The multidimensional prognostic index predicts falls in older people: an 8-year longitudinal cohort study of the osteoarthritis initiative. J Am Med Dir Assoc 21:669-674. https://doi.org/10.1016/j.jamda.2019.10.002

Publisher's Note Springer Nature remains neutral with regard to jurisdictional claims in published maps and institutional affiliations. 